\title{
RUSCOSMIC - THE NEW SOFTWARE TOOLBOX FOR DETAILED ANALYSIS OF COSMIC RAY INTERACTIONS WITH MATTER
}

\author{
E.A. Maurchev, Yu.V. Balabin
}

Polar Geophysical Institute, Apatity, Russia,maurchev@pgia.ru, balabin@pgia.ru

At present, cosmic ray (CR) physics uses a considerable variety of methods for studying CR characteristics of both primary and secondary fluxes. Experimental methods make the main contribution, using various types of detectors, but numerical methods increasingly complement it due to the active development in computer technology. This approach provides researchers with the most extensive information about details of the process or phenomenon and allows us to make the most competent conclusions. This paper presents a concept of the RUSCOSMIC (C) software package based on the GEANT4 toolkit and representing a range of different numerical models for studying CR propagation through medium of different systems (radiation detectors, Earth's atmosphere). The obtained results represent response functions of the main radiation detectors as well as some typical characteristics of secondary CR fluxes. Comparative results also show the operation of the module verification of calculations with experimental data.

Keywords Cosmic rays $\cdot$ Experimental techniques $\cdot$ Numerical methods $\cdot$ Monte Carlo method $\cdot$ Radiation detectors $\cdot$ Particle interaction with matter

\section{INTRODUCTION}

Cosmic rays (CRs) are currently being studied in different ways. Initially these were mainly experimental methods widely used so far. Sounding balloons equipped with detectors are launched [Bazilevskaya et al., 2008], and the worldwide network of neutron monitors is one of the most advanced and reliable ground-based systems for detecting cosmic rays. Data from this network are used to determine characteristics of relativistic solar protons, recorded during events of solar cosmic rays (SCR), by simulating increases in the ground-level background of secondary cosmic rays (GLE events [Vashenyuk et al., 2009; Shea, Smart, 1982]).

From the beginning of computerization, a technique for studying CR propagation through a medium by the Monte Carlo method has been rapidly developed. We have devised a software package RUSCOSMICO, which includes models of detectors of different types as well as a model of primary CR propagation through Earth's atmosphere, to gain information on a cascade of secondary cosmic rays. The software package is based on a toolkit GEANT4 [Agostinelli et al., 2003], which provides classes describing particle interaction with matter, geometry, selection of initial parameters, collection of information on simulation, particle conditions, etc. 
At the first stage of this work, we examined the properties of detection equipment at the cosmic ray station located in the town of Apatity. For this purpose, we worked out software modules representing models of neutron monitor (NM), Geiger counters, scintillation detectors with geometry and properties of materials as close to reality as possible.

When simulating propagation of a neutron flux through a neutron monitor, we found a response function and examined properties of a decelerator and lead generator. Comparison of our results with results of earlier and currently ongoing works showed good agreement between them. Up-to-date interaction cross-sections used in modeling enabled a more detailed study of the response function of the neutron monitor in a range from $10 \mathrm{MeV}$ to epithermal energies. The simultaneously performed modeling of multiplicity in the neutron monitor confirmed the assumption that the phenomenon is caused by a cloud of particles rather than a single particle entering the NM lead generator.

The modeling of scintillation gamma-quantum detectors became necessary because there is an X-ray monitoring system at the CR station, which has been operating for several years already. This system allowed us to reveal X-ray increases during precipitation. GEANT4 was used to calculate response functions of $\mathrm{NaI}$ detectors of different geometry. Of course, such works have also been carried out before, but our study features a modern approach and a completely new concept enabling us to take into account more factors in calculating propagation of particles through a medium.

The second step was to create our own software module to compute propagation of particles of different energies through Earth's atmosphere. This allowed us not only to use continuously updated data for simulation, but also to make it more broadly applicable since we can easily change input parameters of the model itself (to change physical properties of matter, to add various fields, etc.). Then, we calculated propagation of galactic cosmic rays (GCR) through Earth's atmosphere and development of particle cascades during some GLE events. The result is a database containing data on energy spectra of secondary cosmic rays for different heights. These calculations can be used both to measure ionization rate in estimating an equivalent dose and to examine development of cascades during GLE events and to find their new features. The simulation results were compared with the results of similar studies carried out with the PLANETOCOSMICS software package [Maurchev et al., 2011] as well as with experimental balloon data, which show good agreement with our results.

\section{MODEL OF SCINTILLATION NAI DETECTOR}

In detectors of this type, gamma-quantum energy and intensity are determined from secondary charged particles (electrons and positrons), which result from interactions of gamma-quantums with matter. As mentioned in Introduction, all models of the complex are based on the Monte Carlo method and a selected value $x \in\left(x_{1}, x_{2}\right)$ of the normalized probability density function $f(x)$ :

$$
f(x)=\sum_{i=1}^{n} \alpha_{i} f_{i}(x) g_{i}(x)
$$


where $\alpha_{i}>0$ is the probability sample of the normalized probability density function $f_{i}(x)$ and $0 \leq g_{i}(x) \leq 1$. Then, having selected a random integer with a probability proportional to $\alpha_{i}$ and having chosen $x_{0}$ from the distribution $f_{i}(x)$, we can calculate $x=x_{0}$ with a probability of $g_{i}\left(x_{0}\right)$. If the value deviates, the calculation scheme is repeated from the beginning. The detailed mathematical description of the model is too cumbersome and therefore not given in this paper. The above concept was used to design two scintillation detectors with geometrical dimensions $\varnothing 6.3 \times 2 \mathrm{~cm}$ and $\varnothing 15 \times 10 \mathrm{~cm}$. The simulation of propagation of monoenergetic gamma-quantum beams through the scintillation detectors with the above geometry allowed us to estimate gamma-quantum detection efficiency (Figure 1).
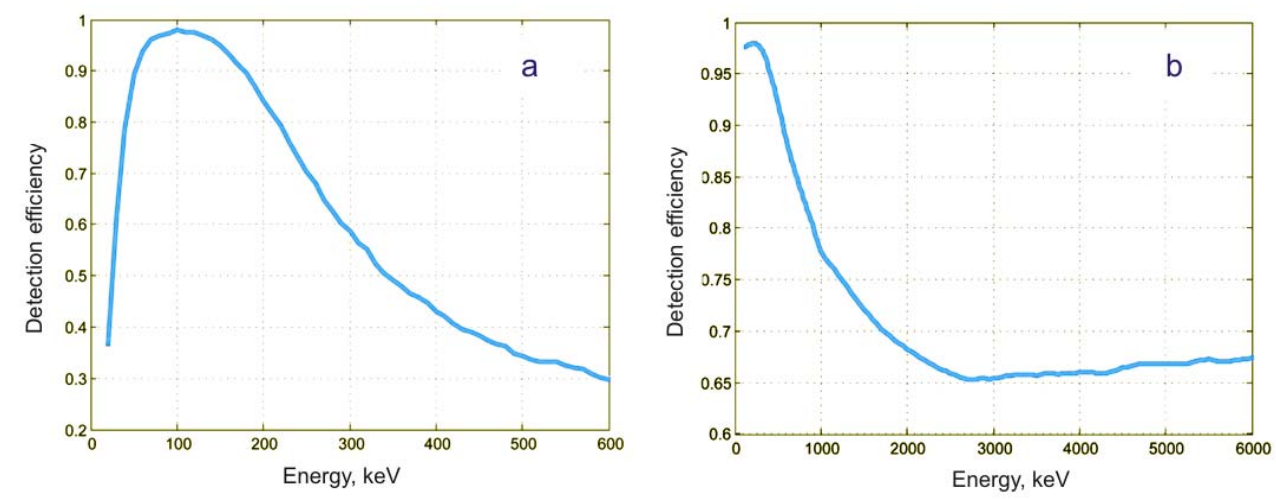

Figure 1. Gamma-quantum detection efficiency obtained from the simulation of interactions of particles with the scintillation detectors of standard sizes $\varnothing 6.3 \times 2(a)$ and $\varnothing 15 \times 10 \mathrm{~cm}(b)$

\section{NEUTRON MONITOR MODEL MOHИTOPA}

Geometrical parameters of the model are identical to the parameters of the real NM, the model is illustrated in Figure 2; the standard tracking is shown in Figure 3. A flux of monoenergetic primary neutrons is incident perpendicular to the upper plane of the detector; the particles are uniformly distributed; the number of particles in the flux is 500000 per energy value. The response function obtained from the simulation is shown in Figure 4.

\section{SIMULATION OF CR PROPAGATION THROUGH EARTH'S ATMOSPHERE}

One of the most complex model in the software package RUSCOSMICC is a model for calculating propagation of primary CR protons through Earth's atmosphere and for studying formations of secondary CR cascades.

The model is based on the concept of the so-called plane geometry when a column of air is separated at a given latitude and longitude, its sizes are specified, and physical parameters of each layer (percentage of masses of constituent elements, density, pressure, and temperature) is calculated using NRLMSISE-00 [Picone et al., 2002]. At the boundary, we determine a model particle source with a preset intensity and spectral characteristic. Figure 5 depicts the spectrum of primary CR pro- 
tons corresponding to the period of maximum solar activity in the absence of SCR. Parameters of secondary CR cascades obtained from the simulation of propagation of primary particles with such energy characteristics through Earth's atmosphere are shown in Figures 6 and 7.

Vertical profiles are usually determined from data acquired by balloons with detectors sensitive to the charged component. The total count can be expressed by the formula

$$
C_{\text {сумм }}=C_{\mathrm{e}^{+} \mathrm{e}^{-}}+C_{\mu^{+} \mu^{-}}+C_{\mathrm{p}}+0.01 \cdot C_{\gamma},
$$

where $C_{\mathrm{e}^{+} \mathrm{e}^{-}}, C_{\mu^{+} \mu^{-}}, C_{\mathrm{p}}, C_{\gamma}$ are counts of electron-positron, muon, and proton components, and gammaquantums respectively.

Accordingly, summing up the vertical profiles determined from the simulation, we can compare them with experimental data for certain periods of time. An example of such verification is shown in Figure 8.

The sum of average and internal errors in the method when simulating the vertical profiles can conveniently be represented as a line width (see Figure 9).

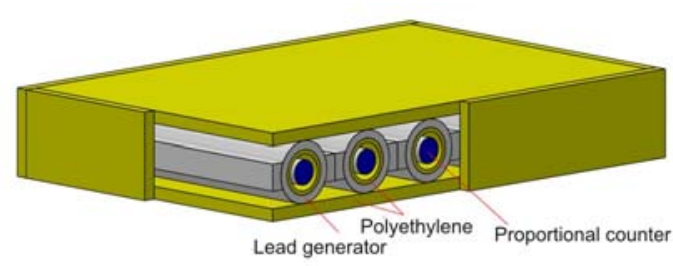

Figure 2. The model of the standard NM, which consists of a polyethylene decelerator, a lead generator, a polyethylene reflector, and proportional ${ }^{10} \mathrm{BF}_{3}$ counters, illustrated to present the main components of the detector

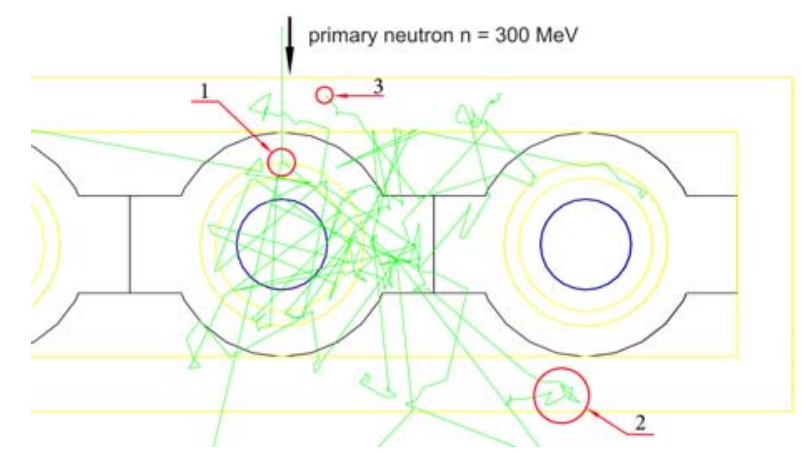

Figure 3. A part of the front projection of the model of one section in the standard NM. A $300 \mathrm{MeV}$ neutron falls on the polyethylene from above. Inelastic collision with lead (we employ the model of intranuclear cascades [Bertini, 1969;. Heikkinen et al, 2003], point “1”) causes neutron multiplication. We can clearly see processes of neutron drift with multiple elastic collisions (point "2"), stop, and absorption in the polyethylene (point "3"). All these processes are calculated using the ENDF/B-VII.1 toolkit [Chadwick et al., 2011]. The process of neutron capture by the counter is not illustrated here, but it should be noted that it is described by the standard reaction $\mathrm{n}+{ }^{10} \mathrm{~B}$ $\rightarrow{ }^{7} \mathrm{Li}+\alpha$ and is simulated also using ENDF / B-VII.1 


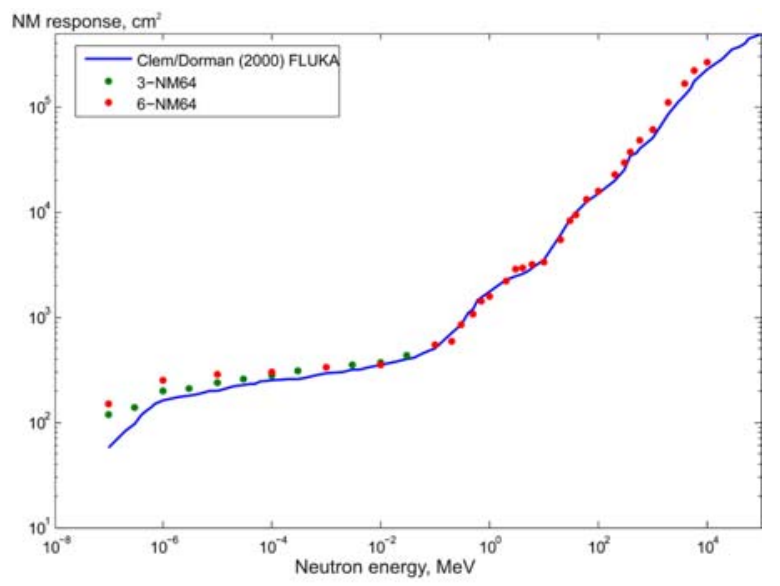

Figure 4. NM response function obtained from the simulation of propagation of monoenergetic neutron fluxes through the detector system. It is compared with a function [Clem, Dorman, 2000] derived previously with the FLUKA toolkit they show close agreement. The difference in the so-called function "tail" $\left(E=10^{-7}-10^{-2} \mathrm{MeV}\right)$ is likely to arise from the use of different models of interactions of low energy neutrons as well as different interaction cross-sections

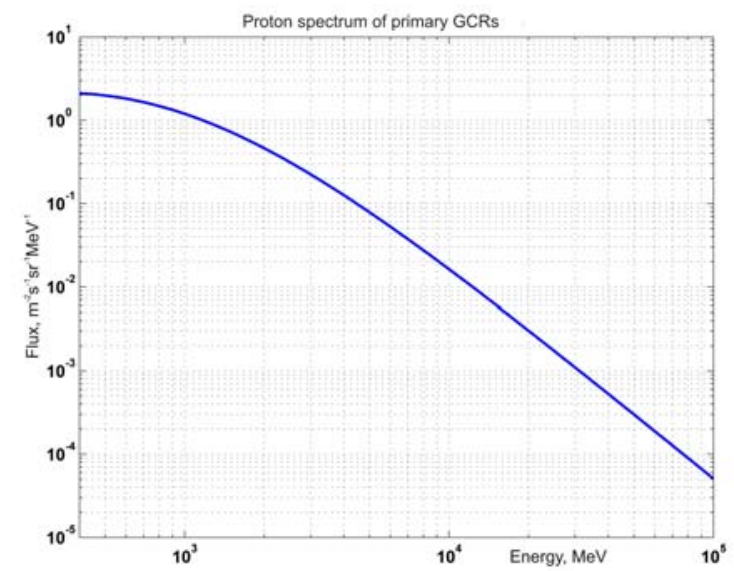

Figure 5. The spectrum of primary GCR protons from 400 to $10^{5} \mathrm{MeV}(\sim 1 \mathrm{GW}$ geomagnetic cutoff rigidity) in the absence of its SCR modulation, used as one of the model particle source characteristics when calculating secondary CR fluxes in Earth's atmosphere
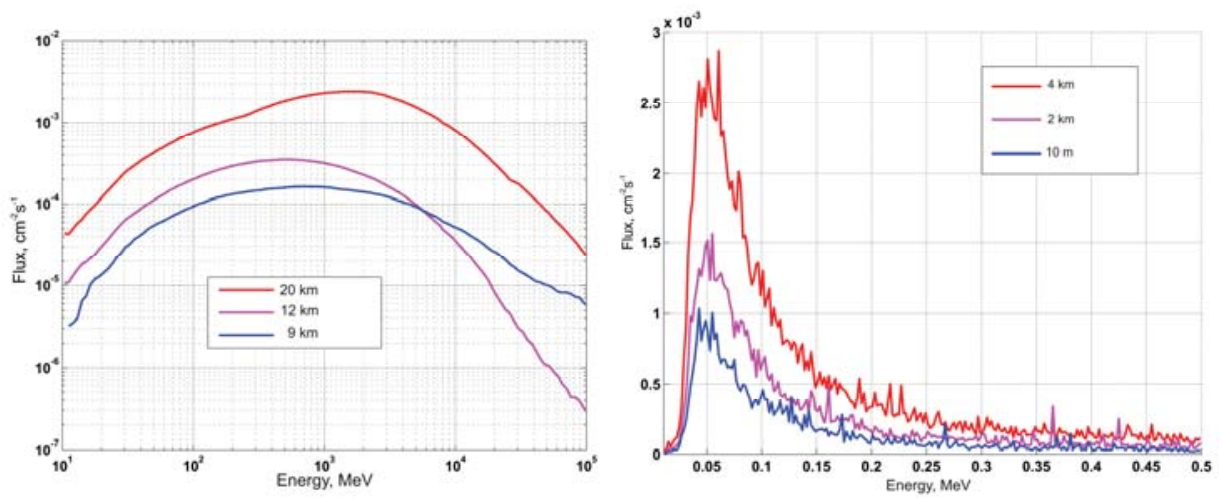

Figure 6. Energy spectra of protons (left) and gamma-quantums (right) of secondary CR obtained from the simulation of propagation of primary CR particles with the spectrum corresponding to the period of maximum solar activity in the absence of its SCR modulation through Earth's atmosphere 

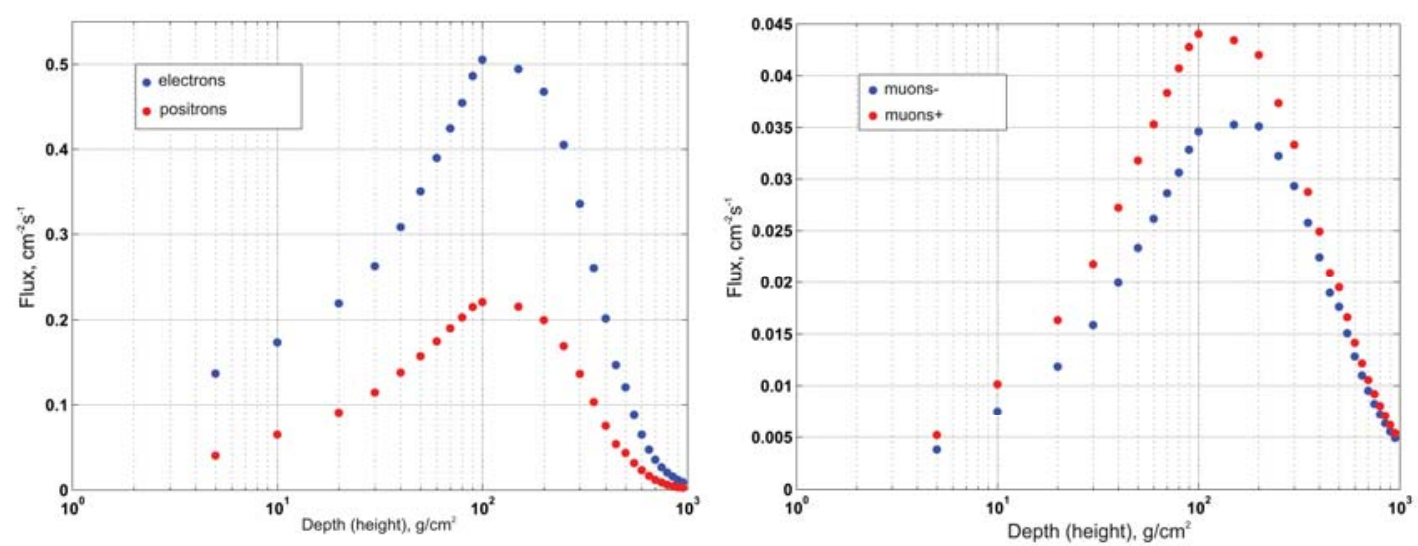

Figure 7. Vertical profiles of electron-positron (left) and muon (right) secondary CR components obtained from the simulation of propagation of primary $\mathrm{CR}$ with the spectrum corresponding to the period of maximum solar activity in the absence of its SCR modulation through Earth's atmosphere
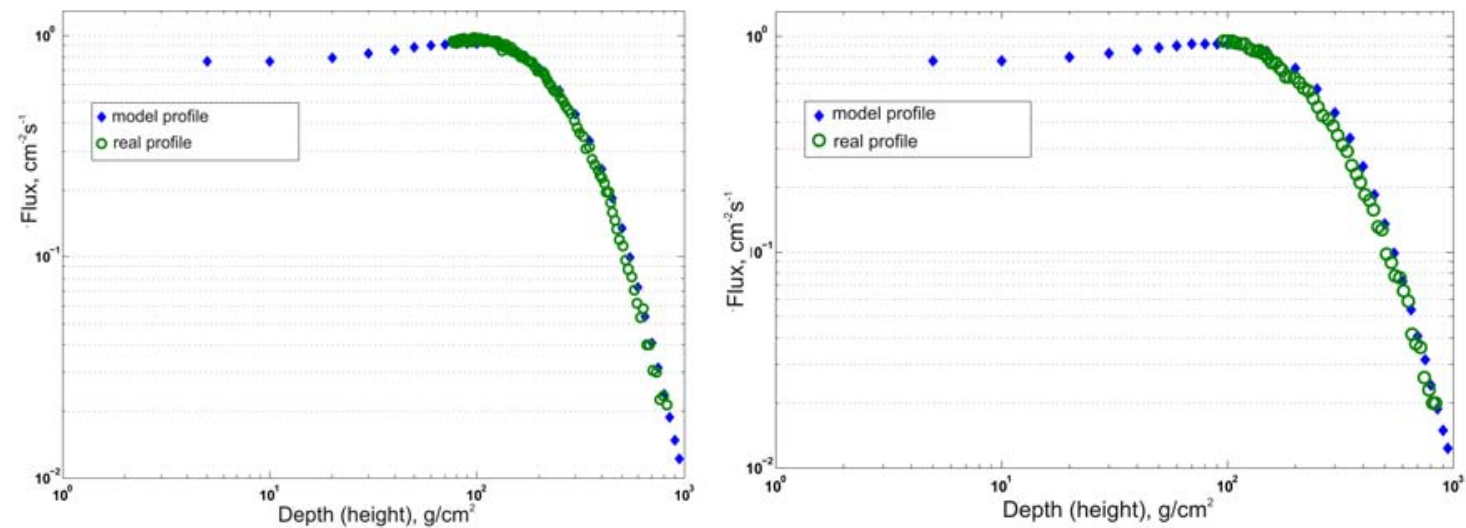

Figure 8. Comparison between experimental data (September 04, 2009 (left) and December 21, 2009 (right)) and data obtained from the simulation of GCR propagation through Earth's atmosphere and processed in accordance with Formula (2)

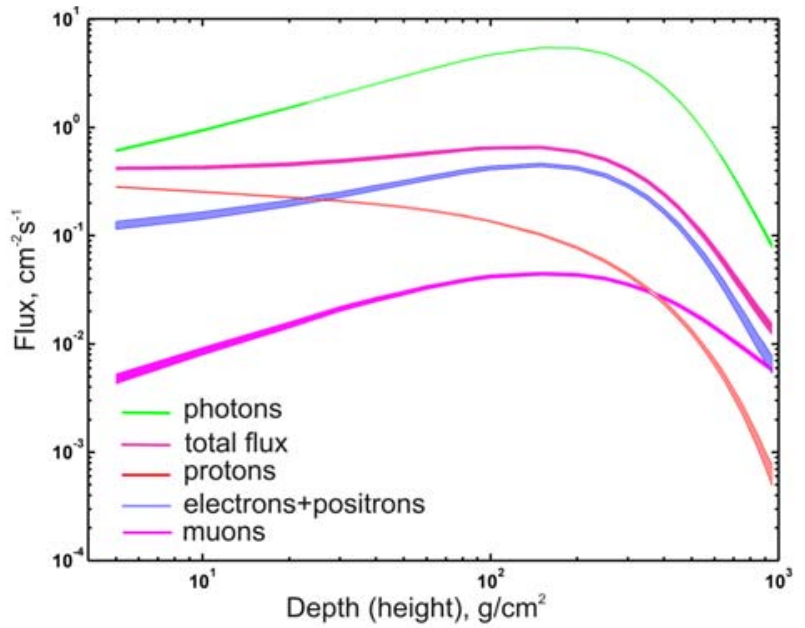

Figure 9. Vertical profiles of particle fluxes presented with a calculation error range, which were obtained from the simulation of GCR propagation through Earth's atmosphere. The line width indicates the total deviation from mean and includes both the standard and internal errors of the method 


\section{CONCLUSION}

In this work, we have carried out a wide range of tasks - from simple simulation of standard detectors, widely used in experiments, to accurate calculations of secondary CR fluxes, generated by propagation of primary CR particles through Earth's atmosphere. We obtained the response function for devices of different types, which then worked well in calibration. A detailed study of characteristics of secondary CR fluxes is a separate task, and its description is beyond the scope of this work. It should be noted, however, that a distinctive property of the calculations of propagation of primary CR protons through Earth's atmosphere is the integration of spectra, obtained in [Vashenyuk et al., 2011], into the model. This allows us to quickly and accurately acquire energy characteristics and vertical profiles of both neutron and charged components (protons, muons, electrons, positrons) not only for ordinary GCR, but also for different instants of SCR events. These results are used to calculate the rate of ionization of Earth's atmosphere under different initial conditions. In addition, we have turned from the simple concept of plane geometry to a more complex global model, which enables us to use in modeling full information on the primary source of particles and to draw maps for different heights. To implement this model fully, it is supplemented with methods to increase the rate of computation, which along with the simulation results will be described comprehensively in subsequent studies.

A great advantage is the fact that the results are to be compared with experimental data, for which purpose there is a huge database accumulated over years. In conclusion, it is worth noting that the very first comparison gave good agreement with a small, within $\sim 10 \%$ from mean, deviation.

\section{REFERENCES}

Agostinelli S., Allison J., Amako K., et al. Geant4 - a simulation toolkit. Nuclear Instruments and Methods in Physics Research. Section A: Accelerators, Spectrometers, Detectors and Associated Equipment . 2003, vol. 506, no. 3, p. 250. DOI: 10.1016 / S0168-9002 (03) 01368-8.

Bazilevskaya G.A. Usoskin I.G., Flückiger E.O., et al. Cosmic ray induced ion production in the atmosphere. Space Sci. Rev. 2008, vol. 137, pp. 149-173.

Bertini H.W. Intranuclear-cascade calculations of the secondary nucleon spectra from nucleon-nucleus interactions in the energy range 340 to $2900 \mathrm{MeV}$ and comparison with experiment. Phys. Rev . 1969, vol. 188, pp. 1711-1730.

Chadwick M.B., Herman M., Obložinský P., et al. ENDF / B-VII.1 Nuclear Data for science and technology: Cross sections, covariances, Fission product yields and decay data. Nuclear Data Sheets. 2011, vol. 112, iss. 12, pp. 2887-2996.

Clem J.M., Dorman L.I. Neutron monitor response function. Space Science Rev. 2000, vol. 93, pp. 335-359.

Heikkinen A., Stepanov N., Wellisch J.P. Bertini intra-nuclear cascade implementation in Geant4. Computing in High Energy and Nuclear Physics. 24-28 March 2003, La Jolla, California. MOMT008.PDF.

Maurchev E.A., Balabin Yu.V., Vashenyuk E.V., Makhmutov V.S. Simulation of the transport of solar protons through the atmosphere in the 13 December 2006 GLE. Physics of Auroral Phenomena: Proc. XXXIV Annual Seminar. Apatity, 2011. pp. 110-113.

Picone J.M., Hedin A.E., Drob D.P., Aikin A.C. NRLMSISE-00 empirical model of the atmosphere: Statistical comparisons and scientific issues. J. Geophys. Res. 2002, vol. 107, no. A12, p. 1468. DOI: 10.1029 / 2002JA009430. 
Shea M.A., Smart D.F. Possible evidence for a rigidity dependent release of relativistic protons from the solar corona. Space Sci. Rev. 1982, vol. 32, pp. 251-271.

Vashenyuk E.V., Balabin Yu.V., Gvozdevsky B.B. Relativistic solar cosmic ray dynamics in large ground level events. Proc. $21^{\text {st }}$ ECRs, Kosice, Slovakia , 9-12 September, 2008. Inst. of Exp. Phys Slovak Academy of Sci Publ., 2009, pp. 264-268.

Vashenyuk E.V. Balabin Yu.V., Gvozdevsky B.B. Features of relativistic solar proton spectra derived from ground level enhancement events (GLE) modeling. Astrophys. Space Sci. Trans. 2011, vol. 7, pp. 459-463. 